Yuanyuan Yang, Li Jin, Zhenyu Zuo*, Chen Yanhui and Chen Fang

\title{
The crystal structure of acetato- $\kappa^{1} O-\{(2-(2-(2-$ aminophenoxy)ethoxy)phenyl)(4-0xo-4- phenylbut-2-en-2-yl)amido-k $\left.{ }^{2} N, N^{\prime}, O\right\}$ copper(II), $\mathrm{C}_{26} \mathrm{H}_{26} \mathrm{CuN}_{2} \mathrm{O}_{5}$
}

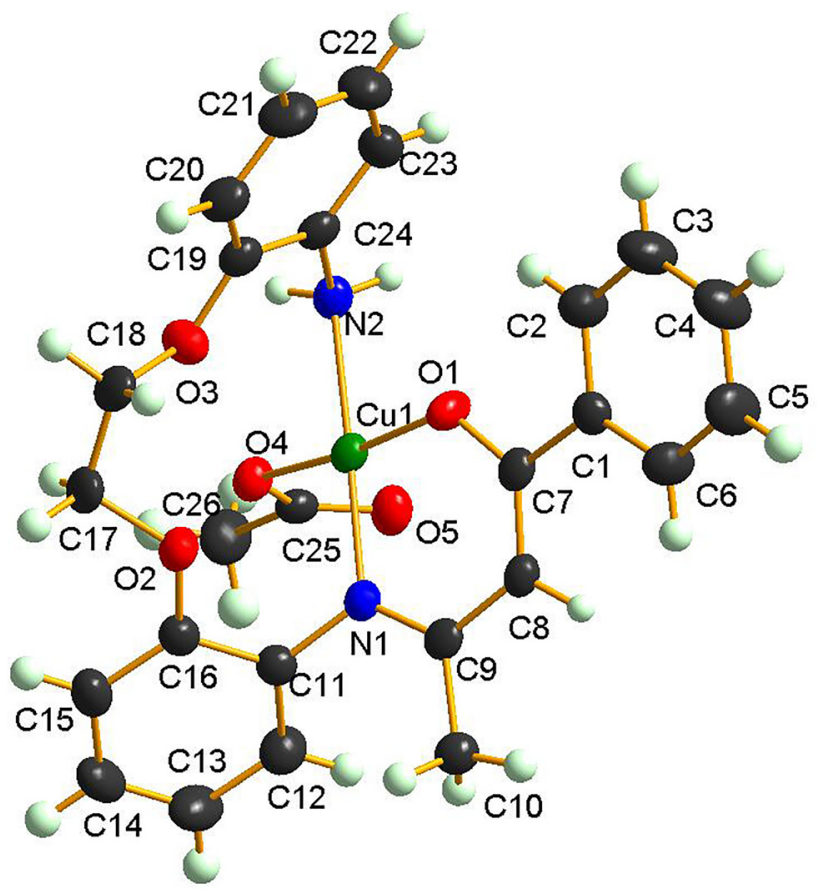

https://doi.org/10.1515/ncrs-2021-0048 Received February 2, 2021; accepted February 18, 2021; published online March 4, 2021

\section{Abstract} $\mathrm{C}_{26} \mathrm{H}_{26} \mathrm{CuN}_{2} \mathrm{O}_{5}$, triclinic, $P=\overline{1}$ (no. 2), $a=8.8090(5) \AA$, $b=11.4984(6) \AA, c=12.3941(7) \AA, \alpha=90.11^{\circ}, \beta=106.017(2)^{\circ}$,

*Corresponding author: Zhenyu Zuo, College of Pharmacy, Shaanxi University of Chinese Medicine, Xi'an 712046, Shaanxi, P. R. China; and Shaanxi Key Laboratory of Basic and New Herbal Medicament Research, Xi'an 712046, P. R. China, E-mail: 123263064@qq.com. https://orcid.org/0000-0003-2519-2574

Yuanyuan Yang and Chen Yanhui, College of Pharmacy, Shaanxi University of Chinese Medicine, Xi'an 712046, Shaanxi, P. R. China, E-mail: 3188131195@qq.com (Y. Yang)

Li Jin, College of Pharmacy, Shaanxi University of Chinese Medicine, Xi'an 712046, Shaanxi, P. R. China; and Shaanxi Key Laboratory of Basic and New Herbal Medicament Research, Xi'an 712046, P. R. China Chen Fang, Shaanxi Institute of Food and Drug Administration and Inspection, Xi’an 712046, Shaanxi, P. R. China
Table 1: Data collection and handling.

\begin{tabular}{|c|c|}
\hline Crystal: & Brown block \\
\hline Size: & $0.24 \times 0.21 \times 0.20 \mathrm{~mm}$ \\
\hline Wavelength: & Mo $K \alpha$ radiation $(0.71073 \AA$ ) \\
\hline$\mu$ : & $0.96 \mathrm{~mm}^{-1}$ \\
\hline Diffractometer, scan mode: & Bruker Apex-II, $\varphi$ and $\omega$ \\
\hline$\theta_{\max }$, completeness: & $25.0^{\circ}, 98 \%$ \\
\hline$N(h k l)_{\text {measured }}, N(h k l)_{\text {unique }}, R_{\text {int }}:$ & $12593,4121,0.025$ \\
\hline Criterion for $I_{\mathrm{obs}}, N(h k l)_{\mathrm{gt}}:$ & $I_{\text {obs }}>2 \sigma\left(I_{\text {obs }}\right), 3696$ \\
\hline$N(\text { param })_{\text {refined }}:$ & 307 \\
\hline Programs: & Bruker [1], SHELX [2], Olex2 [3] \\
\hline
\end{tabular}

$y=100.180(2)^{\circ}, V=1185.95(11) \AA^{3}, Z=2, R_{g t}(F)=0.0315$, $w R_{r e f}\left(F^{2}\right)=0.0869, \mathrm{~T}=293 \mathrm{~K}$.

CCDC no.: 2047023

The molecular structure is shown in the Figure. Table 1 contains crystallographic data and Table 2 contains the list of the atoms including atomic coordinates and displacement parameters.

\section{Source of material}

To the solution of (Z)-3-((2-(2-(2-aminophenoxy)ethoxy) phenyl)amino)-1-phenylbut-2-en-1-one $(0.388 \mathrm{~g} 1 \mathrm{mmol})$ in chloroform $(20 \mathrm{ml})$ was added the solution of $\mathrm{Cu}(\mathrm{AcO})_{2}$ $(0.182 \mathrm{~g}, 1 \mathrm{mmol})$ in chloroform $(20 \mathrm{ml})$ dropwisely. The mixture was stirred for $72 \mathrm{~h}$ to get a blue solution and then filtered. The filter residue was washed with methanol 3 times and recrystallized in chloroform to get crystals $(0.31 \mathrm{~g}$, yield $60.6 \%)$.

\section{Experimental details}

The data were scaled and corrected for absorption using SADABS-2016/2 (Bruker, APEX-II CCD). The hydrogen atoms were placed at calculated positions and refined as riding atoms with isotropic displacement parameters. 
Table 2: Fractional atomic coordinates and isotropic or equivalent isotropic displacement parameters $\left(\AA^{2}\right)$.

\begin{tabular}{|c|c|c|c|c|}
\hline Atom & $x$ & $y$ & $z$ & $U_{\text {iso }} * / U_{\text {eq }}$ \\
\hline $\mathrm{C} 1$ & $0.3724(3)$ & 0.27677 (19) & $-0.26402(17)$ & $0.0288(5)$ \\
\hline $\mathrm{C} 2$ & $0.4435(3)$ & $0.2239(2)$ & $-0.3336(2)$ & $0.0408(6)$ \\
\hline $\mathrm{H} 2$ & 0.5469 & 0.2086 & -0.3045 & 0.049 * \\
\hline $\mathrm{C} 3$ & $.3623(3)$ & $0.1934(3)$ & $-0.4459(2)$ & $0.0517(7)$ \\
\hline H3 & 0.4104 & 0.1567 & -0.4910 & $0.062^{*}$ \\
\hline $\mathrm{C} 4$ & $0.2100(3)$ & $0.2178(3)$ & $-0.4904(2)$ & $0.0515(7)$ \\
\hline $\mathrm{H} 4$ & 0.1554 & 0.1978 & -0.5656 & $0.062^{*}$ \\
\hline $\mathrm{C} 5$ & $0.1401(3)$ & $0.2717(3)$ & $-0.4232(2)$ & $0.0520(7)$ \\
\hline $\mathrm{H} 5$ & 0.0380 & 0.2890 & -0.4534 & $0.062^{*}$ \\
\hline C6 & $0.2192(3)$ & $0.3008(2)$ & $-0.3109(2)$ & $0.0409(6)$ \\
\hline H6 & 0.1695 & 0.3367 & -0.2663 & $0.049^{*}$ \\
\hline $\mathrm{C7}$ & $0.4608(3)$ & $0.30131(18)$ & $-0.14125(17)$ & $0.0273(5)$ \\
\hline C8 & $0.3926(3)$ & $0.35165(18)$ & $-0.06931(17)$ & $0.0278(5)$ \\
\hline $\mathrm{H} 8$ & 0.3030 & 0.3851 & -0.1017 & $0.033^{*}$ \\
\hline C9 & $0.4477(3)$ & $0.35678(17)$ & $0.05037(17)$ & $0.0261(5)$ \\
\hline C10 & $.3326(3)$ & $0.3918(2)$ & $0.10953(18)$ & $0.0319(5)$ \\
\hline $\mathrm{H} 10 \mathrm{~A}$ & 0.2404 & 0.4103 & 0.0549 & $0.048^{*}$ \\
\hline $\mathrm{H} 10 \mathrm{~B}$ & 0.2990 & 0.3275 & 0.1520 & $0.048^{\star}$ \\
\hline $\mathrm{H} 10 \mathrm{C}$ & 0.3852 & 0.4600 & 0.1594 & $0.048^{\star}$ \\
\hline C11 & $0.6218(3)$ & $0.31172(19)$ & $0.22398(17)$ & $0.0290(5)$ \\
\hline C12 & (3) & $2(2)$ & 0.30 & $0.0403(6)$ \\
\hline $\mathrm{H} 12$ & 0.6131 & 0.4742 & 0.2803 & $0.048^{\star}$ \\
\hline$C 13$ & $0.6736(3)$ & $0.3797(3)$ & $0.4172(2)$ & $0.0483(7)$ \\
\hline+13 & 0.6802 & 0.4396 & 0.4697 & $0.058^{*}$ \\
\hline C14 & $0.7041(3)$ & $0.2706(3)$ & $0.45208(19)$ & $0.0469(7)$ \\
\hline H14 & 0.7 & 0.2564 & 0.5282 & $0.056^{*}$ \\
\hline C15 & .6987 (3) & $0.1818(2)$ & $0.3750(2)$ & $0.0413(6)$ \\
\hline H15 & 0.7222 & 0.1089 & 0.3993 & $0.050^{*}$ \\
\hline$c 16$ & $0.6579(3)$ & $0.2021(2)$ & $0.26071(17)$ & $0.0306(5)$ \\
\hline C17 & $0.7410(3)$ & $0.0291(2)$ & $0.2055(2)$ & $0.0422(6)$ \\
\hline $\mathrm{H} 17 \mathrm{~A}$ & 0.6937 & -0.0294 & 0.2489 & $0.051^{*}$ \\
\hline H17B & 0.8500 & 0.0620 & 0.2490 & $0.051^{*}$ \\
\hline C18 & (3) & -0.025 & 0.095 & $0.0398(6)$ \\
\hline $\mathrm{H} 18 \mathrm{~A}$ & 0.7969 & -0.0914 & 0.1069 & $0.048^{\star}$ \\
\hline $\mathrm{H} 18 \mathrm{~B}$ & 0.6306 & -0.0540 & 0.0495 & $0.048^{*}$ \\
\hline$C 19$ & $0.8134(3)$ & $0.05116(19)$ & $-0.06852(18)$ & $0.0309(5)$ \\
\hline $\mathrm{C} 20$ & $0.7568(3)$ & $-0.0542(2)$ & $-0.1327(2)$ & $0.0390(6)$ \\
\hline $\mathrm{H} 2 \mathrm{O}$ & 0.7188 & -0.1229 & -0.1013 & $0.047^{*}$ \\
\hline $\mathrm{C} 21$ & 7576 (3) & $-0.0560(2)$ & $-0.2444(2)$ & $0.0468(6)$ \\
\hline $\mathrm{H} 21$ & 0.7200 & -0.1265 & -0.2881 & $0.056^{*}$ \\
\hline $\mathrm{C} 22$ & $0.8129(3)$ & $0.0447(2)$ & $-0.2913(2)$ & $0.0464(6)$ \\
\hline $\mathrm{H} 22$ & 0.8128 & 0.0422 & -0.3663 & $0.056^{*}$ \\
\hline $\mathrm{C} 23$ & $0.8695(3)$ & $0.1508(2)$ & $-0.22662(19)$ & $0.0369(5)$ \\
\hline $\mathrm{H} 23$ & 0.9070 & 0.2192 & -0.2584 & $0.044^{*}$ \\
\hline $\mathrm{C} 24$ & $0.8698(2)$ & $0.15418(19)$ & $-0.11510(18)$ & $0.0286(5)$ \\
\hline $\mathrm{C} 25$ & $0.9836(2)$ & $0.44472(19)$ & $0.19143(17)$ & $0.0279(5)$ \\
\hline $\mathrm{C} 26$ & $1.1113(3)$ & $0.4787(3)$ & $0.3018(2)$ & $0.0497(7)$ \\
\hline $\mathrm{H} 26 \mathrm{~A}$ & 1.1333 & 0.4086 & 0.3403 & $0.075^{*}$ \\
\hline $\mathrm{H} 26 \mathrm{~B}$ & 1.2077 & 0.5207 & 0.2877 & $0.075^{*}$ \\
\hline $\mathrm{H} 26 \mathrm{C}$ & 1.0741 & 0.5284 & 0.3474 & $0.075^{*}$ \\
\hline N1 & $0.5848(2)$ & $0.32681(15)$ & $0.10547(14)$ & $0.0266(4)$ \\
\hline N2 & $0.9184(2)$ & $0.26092(15)$ & $-0.04383(14)$ & $0.0288(4)$ \\
\hline $\mathrm{H} 2 \mathrm{~A}$ & 0.9417 & 0.3214 & -0.0848 & $0.035^{*}$ \\
\hline $\mathrm{H} 2 \mathrm{~B}$ & 1.0080 & 0.2555 & 0.0094 & $0.035^{\star}$ \\
\hline 01 & $0.59839(18)$ & $0.26859(14)$ & $-0.11157(12)$ & $0.0343(4)$ \\
\hline
\end{tabular}

Table 2: (continued)

\begin{tabular}{lrrrr}
\hline Atom & $\boldsymbol{x}$ & $\boldsymbol{y}$ & $\boldsymbol{z}$ & $\boldsymbol{U}_{\text {iso }}{ }^{*} \boldsymbol{U}_{\text {eq }}$ \\
\hline 02 & $0.64757(19)$ & $0.12037(13)$ & $0.17772(12)$ & $0.0341(4)$ \\
03 & $0.8192(2)$ & $0.06715(13)$ & $0.04194(12)$ & $0.0362(4)$ \\
04 & $0.92797(18)$ & $0.33458(13)$ & $0.17032(12)$ & $0.0314(3)$ \\
05 & $0.93758(19)$ & $0.52259(14)$ & $0.12852(13)$ & $0.0366(4)$ \\
Cu1 & $0.74970(3)$ & $0.29810(2)$ & $0.03286(2)$ & $0.02622(11)$ \\
\hline & & & & \\
Comment &
\end{tabular}

Enaminones can be synthesized by condensation reaction of diketone and primary amine [4]. Their group IV metal complexes were not only used as alternatives to metallocene catalysts but also used in the field of optoelectronic technologies for their large nonlinear responses $[5,6]$. So, the synthesis and property research of novel enaminone complexes were an important orientation in inorganic chemistry $[7,8]$. The molecular structure of the title compound was determined by X-ray single crystal diffraction. There is one complex in the asymmetric unit (see the figure). The bond length of $\mathrm{C} 7-\mathrm{O} 1, \mathrm{C} 9-\mathrm{N} 1$ and $\mathrm{C} 1-\mathrm{C} 2$ are 1.289(3), 1.318(3) and 1.392(3) Å respectively, which are in the normal range [9]. Copper was centered in a distorted tetragonal planar coordination geometry. The sum of the bond angles of O1-Cu1-N1, O1-Cu1-N2, O1-Cu1-O4 and O4-Cu1-N1 are $360.13^{\circ}$, which demonstrated that $\mathrm{O} 1, \mathrm{Cu} 1$, $\mathrm{N} 1, \mathrm{O} 2$ and $\mathrm{N} 1$ are in the same plane.

Author contribution: All the authors have accepted responsibility for the entire content of this submitted manuscript and approved submission.

Research funding: National Key R\&D program of China (2018YFC1706903), Key projects of Shaanxi Provincial Education Department (20JS033).

Conflict of interest statement: The authors declare no conflicts of interest regarding this article.

\section{References}

1. Bruker. APEX2, SAINT and SADABS; Bruker AXS Inc.: Madison, Wisconsin, USA, 2012.

2. Sheldrick G. M. Crystal structure refinement with SHELXL. Acta Crystallogr. 2015, C71, 3-8.

3. Dolomanov O. V., Bourhis L. J., Gildea R. J., Howard J. A. K., Puschmann H. OLEX2: a complete structure solution, refinement and analysis program. J. Appl. Cryst. 2009, 42, 339-341.

4. Shi Y., Hu Y. Syntheses and structures of copper complexes of tetradentate enaminones derived from condensation of benzoylacetone and ferrocenoylacetone with 1,2-bis(2-aminophenoxy) ethane. J. Coord. Chem. 2009, 62, 1302-1312. 
5. Gradinaru J., Forni A., Druta V., Tessore F., Zecchin S., Quici S., Garbalau N. Structural, spectral, electric-field-induced second harmonic, and theoretical study of $\mathrm{Ni}(\mathrm{II}), \mathrm{Cu}(\mathrm{II}), \mathrm{Zn}(\mathrm{II})$, and VO(II) complexes with $\left[\mathrm{N}_{2} \mathrm{O}_{2}\right]$ unsymmetrical Schiff bases of $S$-methylisothiosemicarbazide derivatives. Inorg. Chem. 2007, 46, 884-895.

6. Kanis D. R., Ratner M. A., Marks T. J. Calculation and electronic description of quadratic hyperpolarizabilities. Toward a molecular understanding of NLO responses in organotransition metal chromophores. J. Am. Chem. Soc. 1992, 114, 10338-10357.
7. Biswas A., Drew M. G. B., Ghosh A. Nickel(II) and copper(II) complexes of unsymmetrical tetradentate reduced Schiff base ligands. Polyhedron 2010, 29, 1029-1034.

8. Sarkar B., Bocelli G., Cantoni A., Ghosh A. Copper(II) complexes of symmetrical and unsymmetrical tetradentate Schiff base ligands incorporating 1-benzoylacetone: synthesis, crystal structures and electrochemical behavior. Polyhedron 2008, 27, 693-700.

9. Zuo Z., Lei F., Song X., Wang L. The crystal structure of $\mathrm{N}, \mathrm{N}$-diethyl4,6-bis(naphthalen-2-yloxy)-1,3,5-triazin-2-amine, $\mathrm{C}_{27} \mathrm{H}_{24} \mathrm{~N}_{4} \mathrm{O}_{2}$. Z. Kristallogr. NCS 2018, 233, 1083-1085. 